\title{
Las emociones en la interacción con la tecnología en el profesorado y el alumnado de centros con buenas prácticas $\mathrm{TIC}^{1}$
}

\section{Teachers' and students' emotions in the interaction with technology in schools with codes of ICT best practices}

\author{
JuAn de Pablos POns² \\ jpablos@us.es \\ Mercedes Llorent-Vaquero ${ }^{3}$ \\ mllorent@us.es \\ Universidad de Sevilla, España
}

\section{Resumen:}

El presente trabajo tiene como objetivo conocer las emociones que surgen de la interacción con las TIC en el profesorado y el alumnado de los centros educativos con larga trayectoria y Buenas Prácticas con TIC en Andalucía. Con esta finalidad, se realiza un diseño de corte cuantitativo con un método descriptivo y un diseño tipo encuesta. La muestra es de 18 centros educativos, en los cuales se indaga sobre las emociones del profesorado y el alu-

\begin{abstract}
:
The present study aims to tap into the emotions of teaching staff and students in Spanish schools in the Region of Andalucía when they interact with ICT. The schools chosen for the study had a long-standing experience with ICT programs and a well-defined code of ICT best practices. A quantitative methodological design was used. The sample consisted of 18 schools, in which teachers' and students' emotions were investigated through two
\end{abstract}

1 Este artículo se basa en la investigación "Proyecto TICSE 2.0 - Las políticas de un ordenador por niño en España. Visiones y prácticas del profesorado ante el Programa Escuela 2.0. Un análisis comparado entre Comunidades Autónomas." financiada por el Plan Nacional I+D del Ministerio de Ciencia e Innovación (EDU2010-17037).

2 Dirección para correspondencia (correspondence address):

Juan De Pablos Pons. Dpto. de Didáctica y Organización Educativa. Universidad de Sevilla. Facultad de Ciencias de la Educación. C/ Pirotecnia, s/n. 41013 Sevilla (España)

3 Este trabajo presenta resultados parciales de la tesis doctoral de Mercedes Llorent Vaquero, financiada por el Ministerio de Educación, Cultura y Deporte de España (FPU13/02952). 
Las emociones en la interacción con la tecnología en el profesorado y el alumnado de centros con buenas prácticas TIC

Juan de Pablos Pons y Mercedes Llorent-Vaquero

mnado a través de dos cuestionarios con escalas tipo Likert. Los resultados muestran que las emociones más comunes en el uso de las TIC en ambos colectivos son el bienestar y satisfacción y el orgullo mientras que la disminución de la frustración es la menos frecuente en el uso de las mismas. Asimismo, se identifican algunos elementos asociados a las emociones tanto del profesorado como del alumnado. Entre las conclusiones destaca la necesidad de otorgar relevancia a las emociones que subyacen en la interacción con las TIC tanto en el profesorado como en el alumnado si el objetivo es mejorar los procesos educativos mediados por TIC. Finalmente, el análisis refleja cómo las emociones en el uso de las TIC por parte del alumnado se asocian a factores similares a los que generan una brecha digital en términos de competencia, como son el género, el número de dispositivos que poseen o disponer de Internet en casa, entre otros.

\section{Palabras clave:}

TIC; emociones; profesorado; alumnado; buenas prácticas. questionnaires designed on a Likert scale. The results show that the most common emotions in the use of ICT in both groups are well-being and satisfaction and pride. A decrease in frustration levels is the least reported emotion. Likewise, some elements associated with the emotions of both teachers and students are identified. The conclusions highlight the need to give relevance to the emotions underlying the interaction with ICT in both teachers and students, if the goal is to improve educational processes mediated by ICT. Finally, the analysis reflects how emotions in the use of ICT in students are associated with factors similar to those that generate digital divide in terms of competition, such as gender, the number of electronic devices and Internet connection at home, among others.

\section{Keywords:}

ICT; emotions; teachers; students; good practices.

\section{Résumé:}

Le présent travail a pour objectif de connaître les émotions qui résultent de l'interaction avec les TIC dans le corps enseignant et chez les étudiants des centres éducatifs à longue trajectoire et des bonnes pratiques avec les TIC en Andalousie. À cette fin, une conception de coupe quantitative est réalisée avec une méthode descriptive et une conception de type enquête. L'échantillon est composé de 18 centres éducatifs, dans lesquels les émotions des enseignants et des élèves sont analysées au moyen de deux questionnaires avec des échelles de type Likert. Les résultats montrent que les émotions les plus courantes dans l'utilisation des TIC dans les deux groupes sont le bien-être, la satisfaction et la fierté, tandis que la diminution de la frustration est la moins fréquente dans leur utilisation. De même, certains éléments associés aux émotions des enseignants et des élèves sont identifiés. Parmi les conclusions la nécessité de donner de la pertinence aux émotions qui sous-tendent l'interaction avec les TIC chez les enseignants et les étudiants ressort si l'objectif est d'améliorer les processus éducatifs médiés/transmis par les TIC. Enfin, I'analyse montre comment les émotions liées à I'utilisation des TIC chez les étudiants sont associées à des facteurs similaires à ceux qui génèrent une fracture numérique en termes de concurrence, tels que le sexe, le nombre d'appareils disposant ou ayant Internet chez eux.

\section{Mots-clés:}

TIC; émotions; enseignants; étudiants; bonnes pratiques.

Fecha de recepción: 28-09-2018

Fecha de aceptación: 07-12-2018 


\section{Marco teórico}

\section{Motivación y emociones en el uso de las TIC}

La motivación es un elemento fundamental en el interés, la elección y la constancia en una determinada tarea o proyecto que culmina con la consecución de un objetivo. En este sentido, la motivación condiciona nuestro comportamiento en base al alcance de una meta específica (Mezzalira \& Boruchovitch, 2014). Por tanto, la motivación resulta un componente esencial en la planificación de proyectos tanto profesionales como personales, influyendo en las creencias y valores, y, en consecuencia, en nuestras actitudes y comportamientos (Sanz, 2017; Copriady, 2015).

Una de las claves en cualquier proceso de cambio en los centros educativos es la motivación de todos los agentes implicados. En este sentido, en la integración de las TIC en las aulas, resulta fundamental la motivación tanto del profesorado como del alumnado (Zylka, Christoph, Kroehne, Hartig \& Goldhammer, 2015). Asimismo, de acuerdo con Nolasco y Ojeda (2016) la motivación es el primer paso hacia la alfabetización digital. En este sentido, es un factor determinante en el acceso a la tecnología y en la interacción con la misma.

Específicamente, la motivación en el uso de las TIC está conectada con otros conceptos como el de las emociones en la interacción de las TIC. No obstante, los estudios sobre el papel de las emociones en el uso de las TIC son escasos. En este sentido, resulta relevante el trabajo de Mathrani, Christian y Ponder-Sutton (2016) que asocia la importancia de la diversión en el uso de las TIC, así como la investigación de Zylka, Christoph, Kroehne, Hartig y Goldhammer (2015) que recoge la relación entre el desarrollo del interés y la motivación hacia las TIC y emociones como el sentimiento de competencia o éxito, la autoestima y el sentimiento de autonomía.

Asimismo, la motivación, sobre todo la de carácter intrínseco, se relaciona con la satisfacción de las necesidades humanas como las de autonomía, control o competencia. Esta satisfacción realza el interés por la actividad siendo más proclive a realizarla por su propio beneficio que si viene impuesto exteriormente (Kwon \& Lee, 2017). En esta línea, la motivación, junto con la satisfacción, son los principales incentivos en las experiencias digitales de enseñanza-aprendizaje (Neville, Lam \& Gordon, 2015). 
Las emociones en la interacción con la tecnología en el profesorado y el alumnado de centros con buenas prácticas TIC

Juan de Pablos Pons y Mercedes Llorent-Vaquero

\section{Profesorado}

El factor motivacional del profesorado debe ser considerado para su análisis y reflexión en pro de una mejora integración de las TIC y, en consecuencia, en pro de una mejora de la alfabetización digital del alumnado (Uluyol \& Sahin, 2016). En este sentido, diversos estudios lo contemplan como el mejor medio para alcanzar un objetivo de aprendizaje o formativo entre el propio profesorado, como puede ser el uso pedagógico de las TIC (Karaseva, Pruulmann-Vengerfeldt \& Siibak, 2018). Dicha motivación puede surgir de diferentes fuentes: incentivos, apoyo, beneficios, etc. En este sentido, la motivación intrínseca suele ser el factor principal en el desarrollo de experiencias de aprendizaje mediadas por TIC. No obstante, si la integración de las TIC viene impuesta puede provocar un mal uso de las mismas y un desgaste a nivel emocional del profesorado, burnout (Uluyol \& Sahin, 2016).

De acuerdo con lo expuesto, los profesores con alta motivación se asocian con una actitud de innovación en relación a la tecnología, constatando la relación entre motivación y uso de las TIC (Copriady, 2015). La formación al profesorado es un factor que influye en la motivación de los mismos hacia la integración de la tecnología en las aulas, sin embargo, resulta insuficiente para provocar una transformación real de la mentalidad, un cambio en los valores y las actitudes hacía las TIC en el profesorado (Huertas \& Pantoja, 2016).

De este modo, la motivación de los docentes para el uso de las TIC en las aulas se relaciona con emociones concretas como la satisfacción en su interacción con las TIC y el sentimiento de éxito o realización en el uso de la tecnología. En los casos en los que el profesorado se siente satisfecho en el uso de las TIC el trabajo se convierte en un factor motivante en el que poder integrar la tecnología. Sin embargo, cuando el uso de la tecnología implica una menor satisfacción los docentes no muestran motivación en el uso de las TIC (Chigona, Chigona \& Davids, 2014). Igualmente, resulta interesante el trabajo de Valverde, Fernández y Revuelta (2013) en el que se evidencia la relación entre las buenas prácticas con TIC en los centros educativos y ciertas emociones positivas en el profesorado como son: la mejora de la autoestima, el orgullo o la satisfacción. En contraposición a ello, el mismo estudio muestra cómo las buenas prácticas educativas con TIC se asocian a menores emociones negativas como el estrés, la frustración o el enfado. 
Por tanto, la relación entre el profesorado y las TIC viene determinada en gran medida por las emociones que le suscita la interacción con las mismas. En este sentido, la formación en TIC como su posterior uso en la práctica docente estarán estrechamente relacionados con las emociones que posea el profesorado en relación al uso de la tecnología (Chigona, Chigona \& Davids, 2014; Copriady, 2015; Valverde, Fernández y Revuelta, 2013).

\section{Alumnado}

En relación al alumnado, las TIC se relacionan con un aumento de la motivación de los mismos en los procesos educativos, mostrando una actitud favorable a la integración de la tecnología (Huertas \& Pantoja, 2016). Asimismo, se evidencia una correlación entre la alfabetización TIC de los estudiantes y su motivación hacia el uso de las herramientas digitales (Zylka, Christoph, Kroehne, Hartig \& Goldhammer, 2015). La motivación provoca mayor entusiasmo en los estudiantes, mostrando una mayor predisposición a adquirir nuevo conocimiento y habilidades basadas en su interés y pasión por la tecnología (Karaseva, Pruulmann-Vengerfeldt \& Siibak, 2018).

En esta línea, la motivación del alumnado correlaciona con una serie de constructos cognitivos, de comportamiento y emocionales, siendo las emociones uno de los elementos clave en la motivación hacia su uso. En este sentido, se encuentran estudios que muestran cómo el uso de las TIC se asocia al desarrollo de una mayor seguridad en los propios estudiantes (Zhang \& Liu, 2016) o a una mayor satisfacción (Kim, Kil \& Shin, 2014). Asimismo, existe una relación negativa entre el sentimiento de ansiedad en el uso de las TIC y la motivación, siendo menor la motivación de los estudiantes que sienten ansiedad en su interacción con la tecnología (Zylka, Christoph, Kroehne, Hartig \& Goldhammer, 2015).

De este modo, queda patente la relación entre las emociones, la motivación y el uso de las TIC. Por consiguiente, el alumnado con unas emociones positivas en la interacción con las TIC se encontrará más motivado ante el conocimiento a adquirir o la habilidad a desarrollar (Huertas \& Pantoja, 2016; Kim, Kil \& Shin, 2014; Zylka, Christoph, Kroehne, Hartig \& Goldhammer, 2015). 
Las emociones en la interacción con la tecnología en el profesorado y el alumnado de centros con buenas prácticas TIC

Juan de Pablos Pons y Mercedes Llorent-Vaquero

\section{Método}

\section{Objetivos}

El objetivo de esta investigación es conocer las emociones que surgen de la interacción con las TIC en el profesorado y el alumnado de los centros educativos con larga trayectoria y Buenas Prácticas con TIC en Andalucía. Con este fin, se realiza un diseño de corte cuantitativo con un método descriptivo y un diseño tipo encuesta o survey. Los instrumentos de recogida de datos, elaborados ad hoc, son dos cuestionarios dirigidos a cada uno de los colectivos objeto de estudio: profesorado y alumnado.

De este modo, se establecen los siguientes objetivos específicos:

- Describir las emociones del profesorado en el uso de las TIC.

- Describir las emociones del alumnado en el uso de las TIC.

- Identificar elementos asociados a las emociones del profesorado en el uso de las TIC.

- Identificar elementos asociados a las emociones del alumnado en el uso de las TIC.

\section{Características de la muestra}

De acuerdo con las características del estudio, se realiza un muestreo de tipo no-probabilístico e intencional (Bisquerra, 2004), cuya unidad muestral es el centro educativo. El criterio de selección de los centros es contar con una larga trayectoria en el uso de las TIC y el desarrollo de Buenas Prácticas educativas con TIC, puesto que se pretende analizar la realidad de los centros con este tipo de prácticas. Así pues, se solicita a dos Centros de Profesores de la provincia de Sevilla y uno de Cádiz que seleccionen los centros que cumplen el citado criterio de selección. La muestra final queda constituida por 18 centros educativos $(n=18)$, cuyos datos se resumen en la siguiente tabla (Tabla 1):

Tabla 1

Descripción de la muestra total del estudio

\begin{tabular}{lccc}
\hline & Total & CEIP & IES \\
\hline Centros & 18 & 11 & 7 \\
Profesorado & 136 & 61 & 75 \\
Alumnado & 1882 & 880 & 1002 \\
\hline
\end{tabular}


El profesorado posee una edad media de 43.45 años (DS=7.235), siendo la mínima 27 y la máxima 60. Existe un porcentaje algo mayor de mujeres $(58.9 \%$ ) que de hombres (41.1\%), ejerciendo en un IES el $55.1 \%$ frente a un $44.9 \%$ que lo hace en CEIP. El promedio de años de experiencia docente es de 16.35 años (DS=8.978), con una media de 7.72 años de experiencia docente con TIC (DS=4.585).

En relación al alumnado, la edad oscila entre los 7 y los 20 años, con una media de 12.31 años (DS=2.305). Un 50.8\% son chicos frente a un $49.2 \%$ de chicas. Un $53.2 \%$ son estudiantes de educación secundaria mientras que un $46.8 \%$ cursa educación primaria.

\section{Instrumentos de recogida de datos}

Tal y como se ha comentado previamente, se diseñan dos cuestionarios para la recogida de datos: uno destinado al profesorado y otro al alumnado. Ambos cuestionarios recogen en su parte inicial una serie de datos de identificación del participante (sexo, edad, nivel educativo del centro, etc.) junto con algunas cuestiones específicas destinadas a cada uno de los colectivos:

- Profesorado: años de experiencia docente; años de experiencia docente con TIC; si han sido coordinadores TIC del centro; si han participado en el Plan Escuela TIC 2.0; si participan actualmente en algún grupo de trabajo o de innovación en TIC; si ha realizado algún curso de formación en TIC; y, si se preocupa por reciclarse en el uso de las TIC.

- Alumnado: edad en la que empezó a usar el ordenador, el móvil, la Tablet y las videoconsolas; si tienen Internet en casa; si tienen Ordenador en casa; si le dieron ultraportátil en el colegio; y, el número de aparatos tecnológicos que utiliza en casa.

Seguidamente, se plantean las escalas Likert de 5 valores (1-5), en las que se identifica la frecuencia con la que sienten determinadas emociones al usar las TIC. Específicamente, en relación al profesorado se analiza el estrés, la frustración, el bienestar y satisfacción, la confianza en sí mismo y el orgullo por su trabajo. En cuanto al alumnado, se estudia la curiosidad, el apoyo, el orgullo por su trabajo, la frustración, la confianza en sí mismo, la seguridad en uno mismo, la diversión y el bienestar y satisfacción. 
Ambas escalas se realizan mediante una tabla de especificaciones con la finalidad de avalar la validez de contenido de las mismas. Igualmente, en las dos escalas de medida se comprueba tanto la fiabilidad, con el estadístico Alpha de Cronbach, como la validez de constructo, con la técnica de análisis factorial, logrando los siguientes resultados:

Tabla 2

Fiabilidad y validez de las escalas de medida

\begin{tabular}{|c|c|c|}
\hline Escala & Fiabilidad & Validez de Constructo \\
\hline $\begin{array}{l}\text { Emociones en el uso } \\
\text { de las TIC (profesora- } \\
\text { do) (5 ítems) }\end{array}$ & .924 & $\begin{array}{l}\text { Factor } 1 \text { explica el } 76.6 \% \text { de la varianza } \\
\text { Todos los elementos saturan el factor por } \\
\text { encima del } .70\end{array}$ \\
\hline $\begin{array}{l}\text { Emociones en el uso } \\
\text { de las TIC (alumnado) } \\
\text { (8 ítems) }\end{array}$ & .812 & $\begin{array}{l}\text { Factor } 1 \text { explica el } 43.6 \% \text { de la varianza } \\
\text { Todos los elementos saturan el factor por } \\
\text { encima del } .60\end{array}$ \\
\hline
\end{tabular}

Tal y como expone la Tabla 2, tanto la fiabilidad como la validez de las escalas evidencian la calidad de los instrumentos de medida.

El análisis de los datos expuestos ha sido realizado a través del programa estadístico SPSS en su versión 24. Específicamente, se realizan dos estudios estadísticos: uno descriptivo, en el que se exponen los resultados por ítem de cada una de las escalas de medida (Medias y DT); y otro de contraste, en el que se determinan los aspectos asociados a las dos dimensiones analizadas ( $T$ de Student, ANOVA, H de Kruskal-Wallis y U de Mann-Whitney).

\section{Resultados}

A nivel descriptivo, el profesorado alcanza una media de 2.64 (DS=1.061) respecto a las emociones que surgen de la interacción con las TIC, situándose en torno a la media de la escala al oscilar la misma entre los valores 1 y 5 . Al analizar cada una de los indicadores se obtienen los siguientes resultados (Figura 1). 
Las emociones en la interacción con la tecnología en el profesorado y el alumnado de centros con buenas prácticas TIC Juan de Pablos Pons y Mercedes Llorent-Vaquero

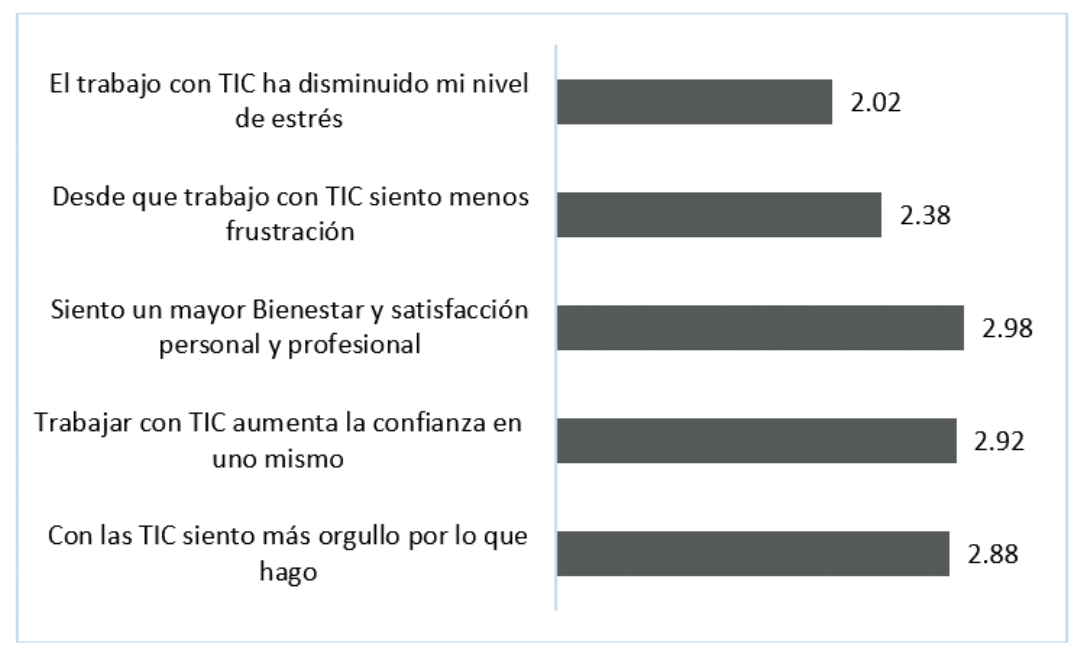

Figura 1. Media de los ítems de la escala sobre "Emociones en el uso de las TIC (profesorado)".

Tal y como se aprecia en la figura anterior, los ítems que alcanzan una mayor valoración por parte del profesorado son los relacionados con el bienestar y la satisfacción, la confianza en uno mismo y el orgullo. En contraposición a ello, la disminución de la frustración, así como del estrés alcanzan menores valores.

En relación a las emociones que surgen de la interacción con las TIC en el alumnado, la media de la escala alcanza el valor 3.47 (DS=0.907), estimándose como una alta valoración al encontrarse en una escala entre 1 y 5 . En la Figura 2 se aprecian las medias de los ítems de la escala.

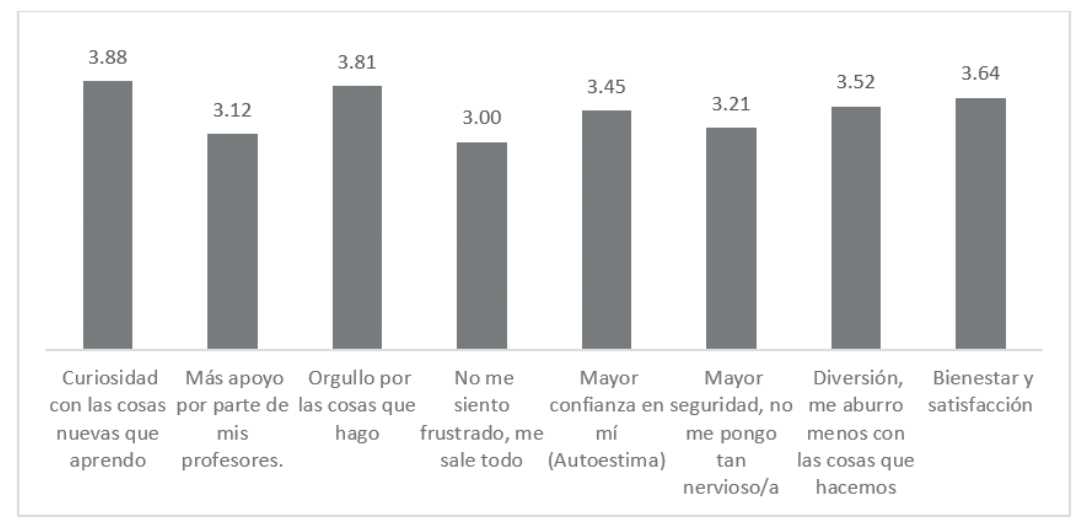

Figura 2. Media de los ítems de la escala sobre "Emociones en el uso de las TIC (alumnado)". 
Los ítems que obtienen una mayor valoración entre el alumnado son los relacionados con la curiosidad, el orgullo, el bienestar y satisfacción y la diversión. Por otro lado, los ítems que alcanzan menor puntuación, aunque se sitúan en valores superiores a la media de la escala (1-5), se asocian a una menor frustración y a un mayor apoyo por parte del profesorado.

A continuación, tras efectuar los correspondientes análisis de distribución normal de la muestra, se exponen los factores asociados a las emociones que surgen de la interacción con las TIC en el profesorado. En la Tabla 3 se muestran, de manera resumida, los resultados obtenidos en cada uno de los contrastes.

Tabla 3

Resultados de contrastes de la dimensión "Emociones en el uso de las TIC (profesorado)" con los datos de identificación

\begin{tabular}{lc}
\hline Nivel educativo & $\begin{array}{c}\text { Emociones en } \\
\text { el uso de las TIC }\end{array}$ \\
Sexo & $.756^{*}$ \\
¿Ha sido coordinador TIC del centro? & $.142^{*}$ \\
¿Participó en el Plan Escuela TIC 2.0? & $\mathbf{. 0 0 0}^{*}$ \\
¿Participa actualmente en algún grupo de trabajo o de & $\mathbf{. 0 0}^{*}$ \\
innovación TIC? & $\mathbf{. 0 2 1}^{*}$ \\
¿Ha realizado algún curso de formación en TIC? & $\mathbf{. 0 3 3}^{*}$ \\
¿Se preocupa por reciclarse en el uso de las TIC? & $\mathbf{. 0 5}^{*}$ \\
¿Ha continuado aplicando la filosofía del Plan Escuela 2.0? & $\mathbf{. 0 0 1}^{*}$ \\
Edad & $.517^{* *}$ \\
Asignatura que imparte & $.221^{* * *}$ \\
Años como docente & $.482^{* *}$ \\
Años usando las TIC & $.372^{* *}$ \\
\hline
\end{tabular}

*Se ha utilizado el estadístico T de Student **Se ha utilizado el estadístico ANOVA ${ }^{* *}$ Se ha utilizado el estadístico $\mathrm{H}$ de Kruskal-Wallis

Como muestra la Tabla 3, la dimensión sobre emociones en el uso de las TIC en el alumnado alcanza diferencias significativas en función de una serie de factores, al rechazar la $\mathrm{H} 0$ de igualdad de medias con un nivel de confianza del 95\%, concretamente en: " $i$ Ha sido coordinador TIC del centro?", "¿Participó en el Plan Escuela TIC 2.0?”, “¿Participa actualmente en algún grupo de trabajo o de innovación TIC", "¿Ha realizado algún 
curso de formación en TIC?", “¿Se preocupa por reciclarse en el uso de las TIC?", y, por último, " ¿Ha continuado aplicando la filosofía del Plan Escuela 2.0?".

Específicamente, las diferencias encontradas en las medias de cada uno de los grupos se muestran de la siguiente manera:

- Coordinador TIC: más emociones en quiénes fueron coordinadores.

- Participación en Plan Escuela 2.0: más emociones en los quiénes participaron en el Plan.

- Participación actual en grupo de trabajo o innovación TIC: más emociones en quiénes participan actualmente en algún grupo de trabajo o innovación TIC.

- Curso de formación TIC: más emociones en quiénes han realizado algún curso de Formación TIC.

- Reciclaje TIC: más emociones en quiénes se preocupan por reciclarse en el uso de las TIC.

- Continuación de la filosofía Plan Escuela 2.0: más emociones en quiénes continúan aplicando la filosofía del Plan.

Respecto a las emociones que muestra el alumnado en el uso de las TIC, tras efectuar los correspondientes análisis de normalidad de la muestra, se realizan una serie de contrastes para observar si existen factores asociados a esta dimensión. La tabla 4 presenta un resumen de los resultados alcanzados en los contrastes:

Tabla 4

Resultados de contrastes de la dimensión "Emociones en el uso de las TIC (alumnado)" con los datos de identificación

Emociones en el uso de las TIC

Nivel educativo

$.000^{*}$

Sexo

$.000^{*}$

¿Tienes Internet en casa?

$.038^{*}$

¿Tienes ordenador en casa?

$.359^{*}$

¿Te dieron ultraportátil en el colegio?

$.000^{*}$

Si te lo dieron, ¿tenías ordenador en casa antes de recibirlo?

$.992 *$

Edad

$.003^{* *}$

Número ordenadores y tablets que utilizan en casa

$.001^{* *}$ 


\begin{tabular}{lc}
\hline & $\begin{array}{c}\text { Emociones en } \\
\text { el uso de las TIC }\end{array}$ \\
\hline Edad en la que empezó a usar el ordenador & $\mathbf{. 0 0 0 ^ { * * }}$ \\
Edad en la que empezó a usar el móvil (Smartphone) & $\mathbf{. 0 0 0}^{* *}$ \\
Edad en la que empezó a usar la Tablet & $\mathbf{. 0 0 0}^{* *}$ \\
Edad en la que empezó a usar la videoconsola & $\mathbf{. 0 0 0}^{* *}$ \\
\hline * Se ha utilizado el estadístico U de Mann-Whitney **Se ha utilizado el esta- \\
dístico H de Kruskal-Wallis
\end{tabular}

Tal y como recoge la Tabla 4, se identifican una serie de factores asociados a las emociones en el uso de las TIC por parte del alumnado. Con un nivel de confianza del $95 \%$ se rechaza la $\mathrm{H} 0$ de igualdad de medias en las siguientes variables: "Nivel educativo", "Sexo", " 2 Tienes Internet en casa?", "¿Te dieron ultraportátil en el colegio?", "Edad", "Número ordenadores y Tablets que utilizan en casa", "Edad en la que empezó a usar el ordenador", "Edad en la que empezó a usar el móvil", "Edad en la que empezó a usar la Tablet", y, finalmente, "Edad en la que empezó a usar la videoconsola".

Concretamente, las diferencias halladas en las medias de los grupos se evidencian de la siguiente forma:

- Nivel educativo: más emociones en el alumnado de Primaria que en el de Secundaria.

- Sexo: más emociones en chicos que en chicas.

- Internet en casa: más emociones en los que sí tienen Internet en casa.

- Ultraportátil: más emociones en los que no recibieron el ultraportátil en el colegio.

- Edad: más emociones en los estudiantes menores de 10 años que en los estudiantes mayores de 15 años.

- Número ordenadores y Tablets que utilizan: más emociones en el alumnado que posee más de 6 dispositivos que en el alumnado que no tiene ninguno.

- Edad Uso Ordenador: más emociones en los que comenzaron a utilizar el ordenador con menos de 5 años que los que comenzaron con más de 10 años.

- Edad Uso Móvil: más emociones en los que empezaron a utilizar el móvil con menos de 5 años que los que empezaron con más de 10 años. 
- Edad Uso Tablet: más emociones en los que comenzaron a utilizar el ordenador entre los 5 y los 7 años que los que comenzaron con más de 10 años.

- Edad Uso Videoconsola: más emociones en los que empezaron a utilizar el móvil con menos de 5 años que los que empezaron entre los 8 y los 10 años.

\section{Discusión y conclusiones}

En relación a los dos primeros objetivos, "Describir las emociones del profesorado en el uso de las TIC" y "Describir las emociones del alumnado en el uso de las TIC", se aprecia una valoración de la dimensión emocional que supera la media en ambas escalas, siendo superior dicha valoración en el alumnado respecto al profesorado. En ambos colectivos el 'bienestar y satisfacción' y 'el orgullo' son las emociones más comunes vinculadas al uso de las TIC, mientras que la disminución de la frustración es la que menos se encuentra presente al utilizar las TIC.

En cuanto al tercer objetivo "Identificar elementos asociados a las emociones del profesorado en el uso de las $\mathrm{TIC}^{\prime \prime}$ se evidencian varios elementos significativos. Las emociones son más frecuentes en los docentes que han participado o participan en planes, programas y proyectos relacionados con TIC. Asimismo, resulta relevante la formación en TIC, tanto la asistencia a cursos como su preocupación por el reciclaje. Por tanto, cabe señalar que esta serie de factores resultan significativos a la hora de establecer que las emociones están presentes, en docentes y estudiantes, con el uso de las TIC. Asimismo, aspectos como el sexo, la edad, los años de experiencia como docente o el tiempo utilizando TIC, no producen diferencias significativas.

Respecto al cuarto objetivo "Identificar elementos asociados a las emociones del alumnado en el uso de las $\mathrm{TIC}^{\prime}$, los resultados muestran una variedad de elementos que aportan diferencias significativas en este sentido. El sexo, la edad, el nivel educativo, si poseen o no Internet en casa, si dispusieron de ultraportátil en la escuela, el número de ordenadores y tablets que tienen en su propio entorno o la edad en la que se iniciaron en el uso de algunos dispositivos, son elementos que marcan diferencias en relación a las emociones en el uso de las TIC. De esta forma, las emociones vinculadas al uso de las TIC en el alumnado, se aso- 
Las emociones en la interacción con la tecnología en el profesorado y el alumnado de centros con buenas prácticas TIC

Juan de Pablos Pons y Mercedes Llorent-Vaquero

cian a factores similares a los que generan una brecha digital en términos de competencia, como son el género, el número de dispositivos, disponer de Internet en casa, etc. (Bartikowskia, Laroche, Jamal \& Yang, 2018; Freiman, Martinovic \& Robichaud, 2018; Pérez-Escoda, Castro-Zubizarreta \& Fandos-Igado, 2016; Schroeder, 2018).

En resumen, tal y como se ha reflejado en la revisión bibliográfica, las emociones desempeñan un papel fundamental en la adquisición de cualquier meta (Hernández-Chérrez \& Tayo, 2017; Reid, Thomson \& McGlade, 2016; Zhang \& Liu, 2016). Por tanto, si el objetivo es mejorar los procesos educativos mediados por TIC, no podemos dejar a un lado las emociones que subyacen en los dos principales agentes involucrados, docentes y estudiantes.

En línea con lo expuesto, respecto al profesorado, tanto la participación en programas y proyectos relacionados con TIC como la formación $\mathrm{TIC}$, se relacionan con mayores emociones en el uso de las TIC. Por tanto, a través de la implementación de políticas, tanto a nivel gubernamental como de centro, se podría fomentar la participación del profesorado en la formación y los proyectos o programas de innovación TIC, cara a aumentar el grado de emociones positivas en el uso de las TIC.

Finalmente, respecto al alumnado, sería conveniente introducirlos en el uso de dispositivos digitales (ordenador, tablet, smartphone o videoconsola) con un apoyo emocional controlado, supervisando el uso que hacen de estas tecnologías. Asimismo, se advierte una brecha digital vinculada al número de dispositivos que poseen en casa. Tal y como muestran los resultados obtenidos, esta brecha podría paliarse a través de la mejora en la dotación de infraestructuras, tal como se realizó en el Plan Escuela 2.0 al dotar de ultraportátiles al alumnado. Este tipo de iniciativas institucionales, que al parecer resulta difícil de mantener en el tiempo, son necesarias ya que permiten generar estrategias a diferentes niveles (macro, meso y micro) que disminuyan las desigualdades. En cuanto a la brecha digital de género existente (Jiménez-Cortés, 2016; Kim, Kil \& Shin, 2014) es importante comenzar desde edades tempranas a fomentar las emociones positivas en el uso de las TIC por parte de las chicas.

Estos aspectos aquí señalados como factores que influyen en los componentes emocionales, y que dan pie a una mejor relación con las tecnologías, en los ámbitos educativos, siempre se producen en un marco pautado por las buenas prácticas TIC. 
Las emociones en la interacción con la tecnología en el profesorado y el alumnado de centros con buenas prácticas TIC Juan de Pablos Pons y Mercedes Llorent-Vaquero

\section{Referencias}

Bartikowski, B., Laroche, M., Jamal, A. \& Yang, Z. (2018). The type-of-internet-access digital divide and the well-being of ethnic minority and majority consumers: A multi-country investigation. Journal of Business Research, 82, 373-380. doi:10.1016/j. jbusres.2017.05.033

Bisquerra, R. (2004). Metodología de la investigación educativa. Madrid: Editorial La Muralla.

Chigona, A., Chigona, W., \& Davids, Z. (2014). Educators' motivation on integration of ICTs into pedagogy: case of disadvantaged areas. South African Journal of Education, 34(3), 01-08.

Copriady, J. (2015). Self- motivation as a mediator for teachers' readiness in applying ICT in teaching and learning. Procedia - Social and Behavioral Sciences, 176, $699-708$. doi:10.1016/j.sbspro.2015.01.529

Freiman, V., Martinovic, D., \& Robichaud, X. (2018). New Faces of Digital Divide and How to Bridge It. En M. Khosrowpour, (Coord.), Encyclopedia of Information Science and Technology, Fourth Edition (pp. 7248-7258). Washington: IGI Global.

Hernández-Chérrez, E. A. \& Tayo, E. (2017). La satisfacción de docentes y estudiantes en una experiencia de aprendizaje apoyada en la tecnología. Revista Publicando, 12(1), 334-350.

Huertas, A. \& Pantoja, A. (2016). Efectos de un programa educativo basado en el uso de las TIC sobre el rendimiento académico y la motivación del alumnado en la asignatura de tecnología de educación secundaria. Educación XX1, 19(2), 229-250, doi: 10.5944/educXX1.14224

Jiménez-Cortés, R. (2016).Ciudadanía digital y bienestar de las mujeres rurales en las redes sociales. RELATEC, Revista Latinoamericana de Tecnología Educativa, 15(2), 81 94. doi:10.17398/1695288X.15.2.81

Karaseva, A., Pruulmann-Vengerfeldt, P. \& Siibak, A. (2018). Relationships between in-service teacher achievement motivation and use of educational technology: case study with Latvian and Estonian teachers. Technology, Pedagogy and Education, 27(1), 33-47, doi:10.1080/1475939X.2017.1339633))

Kim H., Kil, H. \& Shin, A. (2014). An analysis of variables affecting the ICT literacy level of Korean elementary school students. Computers \& Education, 77, 29-38. doi:10.1016/j.compedu.2014.04.009

Kwon, B. \& Lee, J. (2017) What makes a maker: the motivation for the maker movement in ICT. Information Technology for Development, 23(2), 318-335, doi:10.1080/0268 1102.2016.1238816

Mathrani, A., Christian, S., \& Ponder-Sutton, A. (2016). PlaylT: Game Based Learning Approach for Teaching Programming Concepts. Educational Technology \& Society, 19(2), 5-17.

Mezzalira, M. A. \& Boruchovitch, E. (2014). Motivación para leer y comprensión lectora de estudiantes brasileños. Educatio Siglo XXI, 32(2), 119-137. doi:10.6018/j/202191

Neville, V., Lam, M., \& Gordon, C. J. (2015). The impact of eLearning on health profes- 
Las emociones en la interacción con la tecnología en el profesorado y el alumnado de centros con buenas prácticas TIC

Juan de Pablos Pons y Mercedes Llorent-Vaquero

sional educators' attitudes to information and communication technology. Journal of multidisciplinary healthcare, 8, 75-81. doi:10.2147/JMDH.S74974

Nolasco, P. \& Ojeda, M. M. (2016). La evaluación de la integración de las TIC en la educación superior: fundamento para una metodología. RED-Revista de Educación a Distancia, 48(9). doi:10.6018/red/48/9

Pérez-Escoda, A., Castro-Zubizarreta, A., \& Fandos-Igado, M. (2016). La competencia digital de la Generación Z: claves para su introducción curricular en la Educación Primaria. Comunicar, 24(49), 71-79. doi:10.3916/C49-2016-07

Reid, H. J., Thomson, C. \& McGlade, K. J. (2016). Content and discontent: a qualitative exploration of obstacles to elearning engagement in medical students. BMC Medical Education, 16(188). doi:10.1186/s12909-016-0710-5

Sanz, K. (2017). La eliminación del libro de texto y el uso del portafolio en la asignatura de música en ESO: una experiencia para incrementar la calidad del aprendizaje y la motivación del alumnado. Educatio Siglo XXI, 35(1), 9-32. doi:10.6018/j/286201

Schroeder, R. (2018). Towards a theory of digital media. Information, Communication \& Society, 21(3), 1-17. doi:10.1080/1369118X.2017.1289231

Uluyol, C. \& Sahin, S. (2016). Elementary school teachers' ICT use in the classroom and their motivators for using ICT. British Journal of Educational Technology, 47(1), 65-75. doi:10.1111/bjet.12220

Valverde, J., Fernández, M. R. \& Revuelta, F. I. (2013). El bienestar subjetivo ante las buenas prácticas educativas con TIC: su influencia en profesorado innovador. Educación XX1, 16(1), 255-280. doi:10.5944/educxx1.16.1.726

Zhang, D. \& Liu, L. (2016). How Does ICT Use Influence Students' Achievements in Math and Science Over Time? Evidence from PISA 2000 to 2012. Eurasia Journal of Mathematics, Science \& Technology Education, 12(9), 2431-2449. doi:10.12973/ eurasia.2016.1297a

Zylka, J., Christoph, G., Kroehne, U., Hartig, J. \& Goldhammer, F. (2015). Moving beyond cognitive elements of ICT literacy: First evidence on the structure of ICT engagement. Computers in Human Behavior, 53, 149-160. doi:10.1016/j.chb.2015.07.008 